\title{
0797 IMPLEMENTATION OF EVIDENCE-BASED INTERVENTIONS TO PREVENT DROWNING: RESULTS FROM A WHO COLLABORATIVE PROJECT
}

F Mitis*, D Sethi, F Racioppi Correspondence: WHO Regional Office for Europe, Via Francesco Crispi 10, Rome, 00187, Italy

10.1136/ip.2010.029215.797

Introduction 34000 drowning-related deaths are observed yearly in WHO European Region. Rates in low- and middleincome countries are six times higher than in high-income countries.

Methods A questionnaire survey was undertaken as part of a collaborative project between WHO and the European Commission on progress achieved by Member States in implementing the WHO resolution and the European Council Recommendation on the prevention of injuries. Information was used on the implementation of eight evidence-based interventions to prevent drowning. Countries were grouped according to geographical location. Mortalities for drowning were examined by country grouping and correlated with level of implementation of interventions at a subregional level.

Results There were responses from 47 of the 51 Member States with focal persons. Regional median implementation of drowning prevention interventions was $63 \%$ (IQR: $38-75 \%$ ). The average mortality for the Baltic countries is six times higher than that for Nordic countries for both sexes. Implementation was variable across groups: $81 \%$ in Nordic countries, $73 \%$ in southern countries, $70 \%$ in CIS, around $50 \%$ in western, central and southeastern countries, only $8 \%$ in the three Baltic countries. In particular, no interventions were implemented in Lithuania and Latvia, only $25 \%$ in Estonia.

Conclusions An inverse correlation can be observed between the implementation of evidence-based interventions and mortality for drowning in European countries when subregions are considered. The health sector needs to take a leadership role in advocating for the widespread implementation of effective programmes and to engage with other stakeholders in a multisectoral response to prevent drowning. 